Retraction

\title{
Retracted: Flavonoid-Deficient Mutants in Grass Pea (Lathyrus sativus L.): Genetic Control, Linkage Relationships, and Mapping with Aconitase and S-Nitrosoglutathione Reductase Isozyme Loci
}

\author{
The Scientific World Journal \\ Received 7 August 2016; Accepted 7 August 2016 \\ Copyright (C) 2016 The Scientific World Journal. This is an open access article distributed under the Creative Commons Attribution \\ License, which permits unrestricted use, distribution, and reproduction in any medium, provided the original work is properly \\ cited.
}

The Scientific World Journal has retracted the article titled "Flavonoid-Deficient Mutants in Grass Pea (Lathyrus sativus L.): Genetic Control, Linkage Relationships, and Mapping with Aconitase and S-Nitrosoglutathione Reductase Isozyme Loci" [1]. The article was found to contain images with signs of duplication and manipulation in Figures 2 and 3.

\section{References}

[1] D. Talukdar, "Flavonoid-deficient mutants in grass pea (Lathyrus sativus L.): genetic control, linkage relationships, and mapping with aconitase and S-nitrosoglutathione reductase isozyme loci," The Scientific World Journal, vol. 2012, Article ID 345983, 11 pages, 2012. 


\title{
Flavonoid-Deficient Mutants in Grass Pea (Lathyrus sativus L.): Genetic Control, Linkage Relationships, and Mapping with Aconitase and S-Nitrosoglutathione Reductase Isozyme Loci
}

\author{
Dibyendu Talukdar \\ Department of Botany, R.P.M. College, University of Calcutta, Uttarpara, West Bengal, Hooghly 712 258, India \\ Correspondence should be addressed to Dibyendu Talukdar, dibyendutalukdar9@gmail.com
}

Received 25 October 2011; Accepted 25 December 2011

Academic Editors: K. Chakravarty, E. Olmos, and K. Shoji

Copyright (C) 2012 Dibyendu Talukdar. This is an open access article distributed under the Creative Commons Attribution License, which permits unrestricted use, distribution, and reproduction in any medium, provided the original work is properly cited.

\begin{abstract}
Two flavonoid-deficient mutants, designated as fldL- 1 and fldL-2, were isolated in EMS-mutagenized $(0.15 \%, 10 \mathrm{~h}) \mathrm{M}_{2}$ progeny of grass pea (Lathyrus sativus L.). Both the mutants contained total leaf flavonoid content only $20 \%$ of their mother varieties. Genetic analysis revealed monogenic recessive inheritance of the trait, controlled by two different nonallelic loci. The two mutants differed significantly in banding patterns of leaf aconitase (ACO) and S-nitrosoglutathione reductase (GSNOR) isozymes, possessing unique bands in Aco 1, Aco 2, and Gsnor 2 loci. Isozyme loci inherited monogenically showing codominant expression in $\mathrm{F}_{2}$ $(1: 2: 1)$ and backcross $(1: 1)$ segregations. Linkage studies and primary trisomic analysis mapped Aco 1 and fld 1 loci on extra chromosome of trisomic-I and Aco 2, fld 2, and Gsnor 2 on extra chromosome of trisomic-IV in linked associations.
\end{abstract}

\section{Introduction}

Flavonoids are secondary metabolites derived from phenylalanine and acetyl CoA that perform a variety of important functions in plant growth, reproduction, and survival and also serve as important micronutrients in human and animal diets $[1,2]$. The pigmented flavonoid metabolites have been used as phenotypic markers in many model plant species $[3,4]$ and have proven to be an excellent tool to study the genetic, molecular, and biochemical processes $[4,5]$. One of the functional tools in this regard is the genetic characterization of mutants, exhibiting significantly altered flavonoid compounds. A good number of mutants with altered flavonoid levels have been utilized in Arabidopsis, maize, grape, and Petunia to reveal biosynthetic pathway of different flavonoids and their diverse roles [6-10]. Although very rich in flavonoid components [11], no reports are available regarding the genetic analysis of flavonoid mutant in leguminous plants.

Plant flavonoids play pivotal role in protection/tolerance against different types of abiotic stress [12]. Evidences are accumulating about functional interplay between flavonoid metabolism and thiol-based (glutathione/thioredoxin) antioxidant defense system in plants during stress response $[13,14]$, where nitric oxide (NO) functions as signaling molecule [15]. The enzyme aconitase (ACO) is known to be responsible in iron homeostasis and in regulating resistance to oxidative stress [16], but its activity is inhibited by NO [17]. On the other hand, the S-nitrosoglutathione reductase (GSNOR) activity has been described to be associated with the enzyme glutathione-dependent formaldehyde dehydrogenase [18]. GSNOR uses GSNO as its substrate which is formed by the reaction of reduced glutathione with NO molecule. GSNOR is extremely important in maintenance and turnover of cellular $\mathrm{NO}$ pool and modulation of hormonal response such as jasmonic acid and salicylic acid, responsible for alteration of stress-induced phenylpropanoid pathway $[14,19]$.

Grass pea (Lathyrus sativus L.), an annual winter legume crop, possesses high level of bioactive compounds including flavonoids [20]. The potential of this hardy crop has been extensively utilized in recent years through isolation and genetic analysis of novel mutants for plant habit [21], flower and seed coat colour [22, 23], pod indehiscence [24], seed size [25], and so forth. Some of these mutant lines are now being tested for their fitness to different abiotic stresses 
including salinity $[26,27]$ and arsenic [28], and very recently, a novel ascorbate-deficient mutant has been detected [29]. Linkage mapping and chromosomal assignment of desirable mutations are now being accomplished through establishment of a functional cytogenetic stocks including aneuploids [30, 31], polyploids [32], and translocation lines [33, 34]. Perusal of literature cites only limited information regarding inheritance and linkage association of morphological, biochemical (isozyme), and other molecular markers in grass pea [35]. Although isozyme markers are widely used in gene mapping of different crops and have advantages over other markers due to their codominant expression, lack of sufficient number of polymorphic isozymes loci possesses problems in existing germplasms of grass pea [36]. Creation of additional variability in esterase and root peroxidase isozyme systems through induced mutagenesis has recently been successfully explored in dwarf mutant population of this crop, and genetic control of their allozyme variants has been studied [37]. During screening of desirable mutations in EMS-mutagenized population, two variant plants with white flower color was isolated. The mutants were later found to be highly deficient in total flavonoid content in their leaves. Despite immense importance of flavonoids in legume crops and its relation with enzymes involved in stress responses, no reports in these regards are available in grass pea.

Keeping all these in mind, a genetic approach has been taken to investigate the basis of flavonoid deficiency in the preset materials of grass pea and its association with isozymes of ACO and GSNOR enzymes. The main objectives of the present work are to (1) trace the mode of inheritance of flavonoid deficiency and the zymogram phenotypes of both enzymes, (2) investigate the segregation pattern and linkage associations between different isozymes loci and loci controlling flavonoid deficiency, and (3) ascertain their possible chromosome location through primary trisomic analysis.

\section{Methods}

2.1. Plant Materials. Altogether eleven parents have been used in the present study of which four were diploid $(2 n=$ $14)$ and rest seven were primary trisomic $(2 n+1=15)$ types. Among the diploid parents, two varieties "BioL-212" and "Hooghly Local" were used as mother control throughout the experiment. Fresh and healthy seeds of these two varieties presoaked with water $(6 \mathrm{~h})$ were treated with freshly prepared $0.15 \%$ aqueous solution of EMS (Sigma-Aldrich) for $10 \mathrm{~h}$ with intermediate shaking at $25 \pm 2^{\circ} \mathrm{C} . \mathrm{M}_{1}$ seeds were sown treatment-wise in completely randomized block design as reported earlier [25]. Two variant plants showing white flowers and absence or modified stipule morphology were distinguished from usual occurrence of blue flower and typical papilionaceous stipules in EMS-treated $\mathrm{M}_{2}$ progeny. During screening of antioxidant activities of different mutant lines, these two plants exhibited abnormally low foliar flavonoid contents. The levels were again confirmed at $\mathrm{M}_{3}$ generation, and on the basis of stipule characters the
TABLE 1: Total foliar flavonoid contents ( $\mathrm{mg} \mathrm{g}^{-1}$ extract) in aqueous and ethanol extract of grass pea (Lathyrus sativus L.) mutants (fld $L-1$ and fld L-2) and mother plants (BioL-212 and Hooghly Local).

\begin{tabular}{lcc}
\hline Genotype & Aqueous extracts & Ethanol extract \\
\hline BioL-212 & $160.55 \pm 3.6$ & $354.37 \pm 3.9$ \\
Hooghly & $148.59 \pm 3.2$ & $346.40 \pm 3.1$ \\
Local & $150.53 \pm 3.2$ & $70.13 \pm 2.2^{*}$ \\
fld $L-1$ & $30.77 \pm 1.4^{*}$ & $337.27 \pm 2.9$ \\
fld L-2 &
\end{tabular}

${ }^{*}$ Significantly different from mother plants at $P<0.05$

progeny of the two plants was primarily designated as fld L-1 (flavonoid-deficient Lathyrus type 1 mutant, white flower, estipulate) and fld L-2 (flavonoid deficient Lathyrus type 2 mutant, white flower, linear-acicular stipule). Both the mutants bred true for their phenotypes, and no significant change in leaf flavonoid content was found in $\mathrm{M}_{3}$ generation. Chromosome location of different loci was performed by utilizing a set of primary trisomics, isolated and characterized earlier in grass pea $[30,38]$.

2.2. Determination of Total Flavonoid Content. Total flavonoid content from leaves of mutants and their control varieties were determined spectrophotometrically in both ethanol and aqueous extracts, based on the formation of a flavonoidaluminium complex [39]. An amount of $2 \%$ ethanolic $\mathrm{AlCl}_{3}$ solution $(0.5 \mathrm{~mL})$ was added to $0.5 \mathrm{~mL}$ of sample. After $1 \mathrm{~h}$ at room temperature, the absorbance was measured at $420 \mathrm{~nm}$. A yellow color indicated the presence of flavonoids. Extract samples were evaluated at a final concentration of $0.1 \mathrm{mg} \mathrm{mL}^{-1}$. Total flavonoid contents were calculated as rutin $\left(\mathrm{mg} \mathrm{g}^{-1}\right.$ of extract) (Table 1$)$.

2.3. Isozyme Analysis: Gel Electrophoresis and Nomenclature. Horizontal 10\% starch-gel (Sigma) electrophoresis was carried out to analyse the banding profile of aconitase (ACO, EC 4.2.1.3) in mutants $\left(\mathrm{M}_{4}\right)$ and control varieties and trisomic lines. Crude extracts were prepared by macerating young leaf tissues of 4-d-old seedlings in ice-cold extraction buffer containing 20\% sucrose, 5\% PVP-40, $0.1 \mathrm{M} \mathrm{KH}_{2} \mathrm{PO}_{4}$, $0.05 \%$ triton X-100 (Sigma), and $14 \mathrm{mM}$ 2-mercaptoehanol (Sigma) at pH 7.0. Triton X-100 and 2-mercaptoehanol were added just before use. After extraction, sample was stored at $-20^{\circ} \mathrm{C}$ for future use. ACO isozymes were separated using the electrode and gel buffer system ( $\mathrm{pH}$ 6.5) of Cardy et al. [40]. Bands of ACO systems were stained according to the recipes (0.1 M Tris-HCL, $\mathrm{pH} 8.0$, cis-Aconitic acid, $\mathrm{MgCl}_{2}$, Isocitrate dehydrogenase, MTT, PMS, and NADP) of Cardy and Beversdorf [41]. For GSNOR (EC 1.2.1.1) activity, native PAGE was done using 6\% acrylamide gels in TRIS-boricEDTA buffer ( $\mathrm{pH}$ 8.0). For staining of GSNOR activity, gels were soaked in $0.1 \mathrm{M}$ sodium phosphate, $\mathrm{pH} 7.4$, containing $2 \mathrm{mM} \mathrm{NADH}$ for $15 \mathrm{~min}$ in an ice bath. Excess buffer was drained, and gels were covered with filter paper strips soaked in freshly prepared $3 \mathrm{mM}$ GSNO. After $10 \mathrm{~min}$, the filter paper was removed, and gels were exposed to UV-light and 
analysed for the disappearance of the NADH fluorescence, indicating GSNOR activity [42].

Based on the observed variations, isozyme bands were assigned to putative loci following the principles of Weeden [43]. The isozymes were designated as all letters capitals (ACO and GSNOR) but the loci controlling these two isozymes had only the first letter capitalized and presented in italics (Aco and Gsnor). When two or more isozymes, coded by different loci in an enzyme, were visualized on gel, they were numbered sequentially according to their mobility relative to the anode with the most anodal isozyme being number one, and subsequent isozymes were assigned sequentially higher numbers. Likewise, the most anodal allele producing allozyme (fastest variant) of a particular locus was termed as "a" and progressively slower forms "b", "c", and so on. Only clearly visible bands for both enzyme systems were scored in the present study.

2.4. Inheritance and Linkage Analysis. Inheritance and linkage of loci controlling flavonoid deficiency and different isozymes were traced in segregating populations of $\mathrm{F}_{2}$ and backcross generations derived from single locus as well as joint segregation of two loci in different cross-combinations. Following two generations of selfing, intercrosses including reciprocals were made among control varieties and the mutant lines $\left(\mathrm{M}_{4}\right)$ to raise $\mathrm{F}_{1}$ and, subsequently, backcross $\left(\mathrm{BC}_{1}\right)$ and $\mathrm{F}_{2}$ progenies (Table 2). Measures were taken at every stage from sowing to harvesting to prevent any type of outcrossing pollination and intermixing. For allelism test, intercrosses were made among fld $L-1$ and fld $L-2$. Chi-square test was employed to test the goodness of fit between observed and expected values for all crosses (Table 2). Zymogram phenotypes of both ACO and GSNOR were studied in selfed and intercrossed ( $F_{2}$ and backcross) progenies of different parents.

Linkage associations of the segregating isozyme markers along with flavonoid deficiency trait were examined for pair-wise combinations of different isozyme loci and also between pairs of isozyme loci and loci controlling flavonoid deficiency for the expected ratio of $1: 2: 1: 2: 4: 2: 1: 2: 1$ and $3: 1: 6: 2: 3: 1$, respectively, in the $F_{2}$ progeny. Testcross population was raised by crossing $F_{1}$ plant with the parent showing comparatively slow moving allozymes in case of isozyme loci and with recessive lines in segregation of flavonoid deficiency. Chi-square test was employed to test the goodness of fit, and significant deviation from the expected ratio was considered as linkage between the markers. Recombination fraction $(r)$ was calculated from testcross data and was converted to map distance in centiMorgans (cM) through Kosambi's mapping function [44]. Data from different families was pooled when homogeneous for analysis (Table 3)

2.5. Mapping Flavonoid-Deficient Mutant and Isozyme Loci by Primary Trisomic Analysis. The seven primary trisomic types were crossed as female parent with the four different homozygous diploid genotypes (two controls, two mutants), and $F_{1}$ population was obtained in each case. The trisomic
$F_{1}$ plants could be readily identified at early seedling stage on the basis of their specific leaflet phenotypes [30]. Trisomic $F_{1}$ plant was self-fertile and subsequently selfed to obtain $\mathrm{F}_{2}$ progeny and also backcrossed to the respective diploid parent to produce $\mathrm{BC}_{1}$ population. In the segregating $\mathrm{F}_{2}$ progeny, banding patterns were analysed by means of chisquare test for a fit to a normal disomic ratio. Significant deviation from the expected disomic ratio of $1: 2: 1$ in $\mathrm{F}_{2}$ and $1: 1$ in $\mathrm{BC}_{1}$ was further tested with the expected trisomic ratios of $4: 4: 1$ in $\mathrm{F}_{2}, 2: 1$ in $\mathrm{BC}_{1}$ in diploid portion and $2: 7: 0\left(\mathrm{~F}_{2}\right)$ in trisomic portion of the progeny to locate possible chromosome/s, bearing gene/s of different isozyme loci (Table 4). Necessary cytological confirmation of trisomy was performed at meiosis-I following Talukdar and Biswas [30]. To save space, only segregation of trisomics carrying concerned loci has been presented in Table 4.

2.6. Statistical Analysis. Total flavonoid contents in leaves of mother and mutants are presented as mean \pm standard error (SE) with 20 plants in each of the four genotypes. Significant differences between mother and mutant plants for total flavonoids were determined by simple " $t$-test." A probability of $P<0.05$ was considered significant.

\section{Results}

3.1. Total Flavonoid Contents and Morphology of Mutant and Mother Plants. Total flavonoid contents as determined in aqueous and ethanol extract in leaves of mutant and mother leaves were significantly $(P<0.05)$ different. Leaves of both the mutants contained total content $\left(\mathrm{mgg}^{-1}\right.$ extract) only $20 \%$ of mother plants (Table 1). However, flavonoid content was nearly normal in aqueous extract of fldL-1 mutant, but reduced by about 5 -fold in ethanol extract. In contrast, flavonoid content reduced marginally in ethanol extract of fldL-2 leaves but had reduced by nearly 5 -fold in aqueous extract (Table 1).

Both the mutants produced characteristic white flower and modification in stipule characters. While $f l d L-1$ was completely estipulate, a linear-acicular type of stipule was observed in fldL-2 plants. Furthermore, fldL-1 showed normal pollen fertility (98.77\%) like mother plants, while it reduced $(66 \%)$ in fldL-2 plants. Root formation in both mutants, however, was quite normal.

3.2. Inheritance and Allelic Relationship of Gene/s Controlling Flavonoid Deficiency. Reciprocal crosses between fldL-1 as well as fldL-2 and mother control varieties yielded $\mathrm{F}_{1}$ plants with normal level of flavonoids (Table 2). Segregation of normal and flavonoid-deficient plant type showed good fit to $3: 1$ in $\mathrm{F}_{2}$ and $1: 1$ in backcross (Table 2). Flavonoid content from leaves of every genotype was tested and verified with parents. The recessive mutants recovered in $\mathrm{F}_{2}$ generation of the above two crosses were also self-pollinated, and in $\mathrm{F}_{3}$ all the 210 plants exhibited only marginal variations in total flavonoid content compared with their respective parents (data not in table). 
TABle 2: Single locus segregation of fld 1 and fld 2 mutations, two aconitase (Aco \& 2), and S-nitrosoglutathione reductase 2 (Gsnor 2) isozyme loci in $\mathrm{F}_{2}$ and backcross $\left(\mathrm{BC}_{1}\right)$ populations of different intercrosses among four parents in Lathyrus sativus L. ${ }^{\mathrm{a}} \mathrm{FF}-\mathrm{Homozygote}$ of fast alleles, SS-Homozygote of slow allele, FS-Heterozygotes. *, **, and *** consistent with $1: 2: 1,1: 1$, and $3: 1$ ratios, respectively, at $5 \%$ level of significance, ${ }^{++}$parent/s showing slow allozyme used in testcross with $\mathrm{F}_{1}$ and pooled data of several crosses presented.

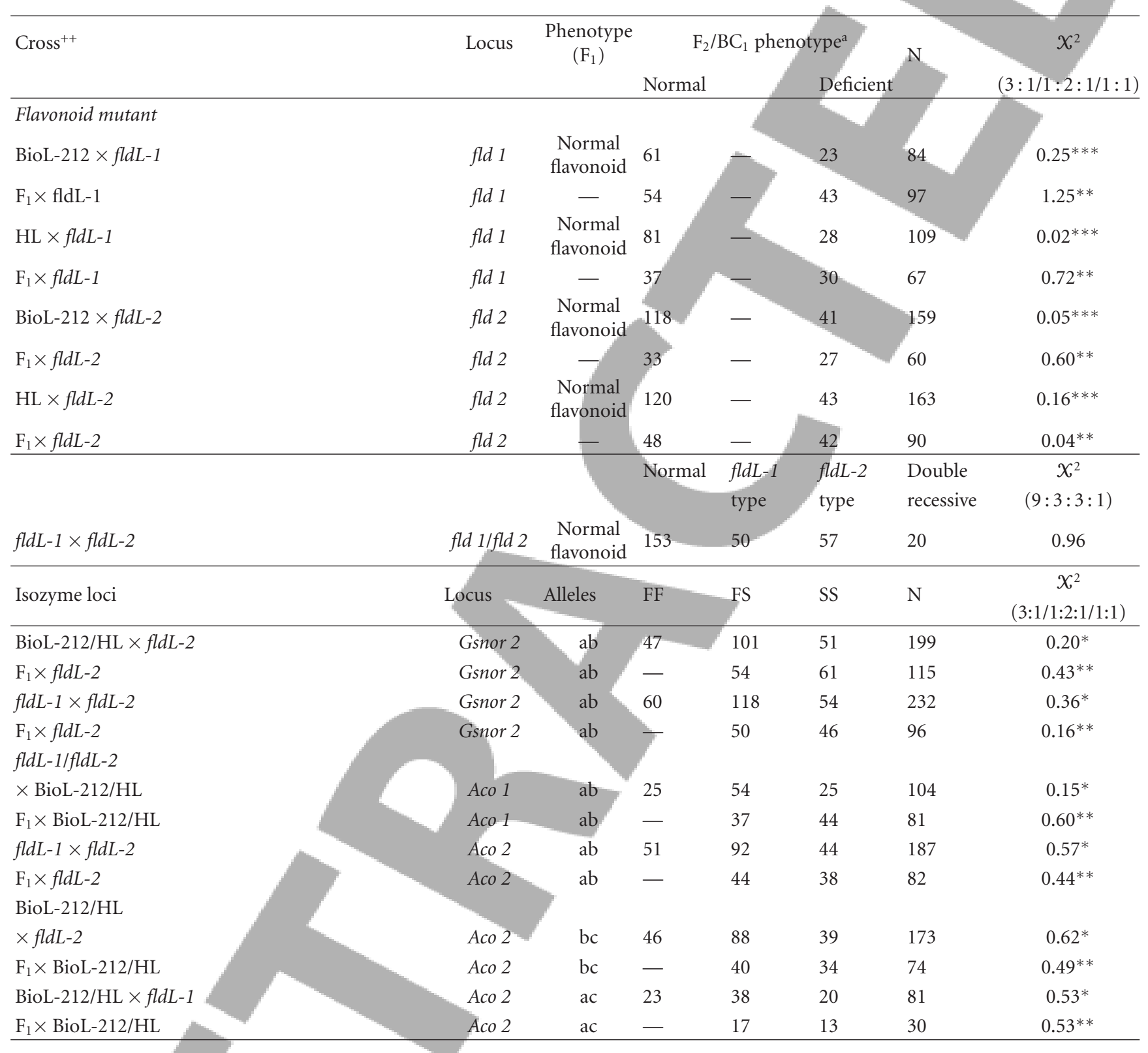

In order to study the allelic relationships of genes governing flavonoid deficiency in grass pea, fldL-1 and fldL2 were reciprocally crossed. All the $\mathrm{F}_{1}$ plants derived from the crosses contained normal flavonoid level like mother control plants. In $\mathrm{F}_{2}$, four types of plants: normal type, fldL1 , fldL-2, and a variant type appeared in the progeny showing good fit to $9: 3: 3: 1$ ratio (Table 2 ). Gene symbols of Fld for normal type and fld 1 and fld 2 for fldL-1 and fldL2 were assigned, respectively. The normal plant type, thus recovered, manifested usual phenotypes such as blue flower, papilionaceous stipules, and normal level of foliar flavonoids. The variant plant type exhibited extreme reduction in total flavonoid contents, containing only $10 \%$ of that in mother control, and this feature was accompanied with reduced root length, absence of stipules, abnormal elongation of leafless stem, and much higher pollen sterility $(79.33 \%)$ than either of its parents.

\subsection{Inheritance of Isozyme-Banding Pattern in Selfed and Intercrossed Progenies}

3.3.1. ACO. Two mutant lines and the control varieties bred true for their respective single-banded phenotypes in successive selfed generations $\left(\mathrm{M}_{2}-\mathrm{M}_{4}\right)$. Two zones of enzyme activity were conspicuous of which the most anodal 
TABLE 3: Joint segregation of pairs of four isozyme loci and fld 1 and fld 2 genes exhibiting significant deviations from expected $\mathrm{F}_{2}$ and backcross $\left(\mathrm{BC}_{1}\right)$ ratios of random assortment in Lathyrus sativus $\mathrm{L} .{ }^{a} \mathrm{H}_{1}$-Heterozygous for alleles at "X" locus, H-heterozygous for alleles at "Y" locus. $r$-recombinant value. ${ }^{*},{ }^{*}$, and ${ }^{* * *}$ significant at $5 \%$ level for $3: 1: 6: 2: 3: 1,1: 1: 1: 1$, and $1: 2: 1: 2: 4: 2: 1: 2: 1$, respectively.

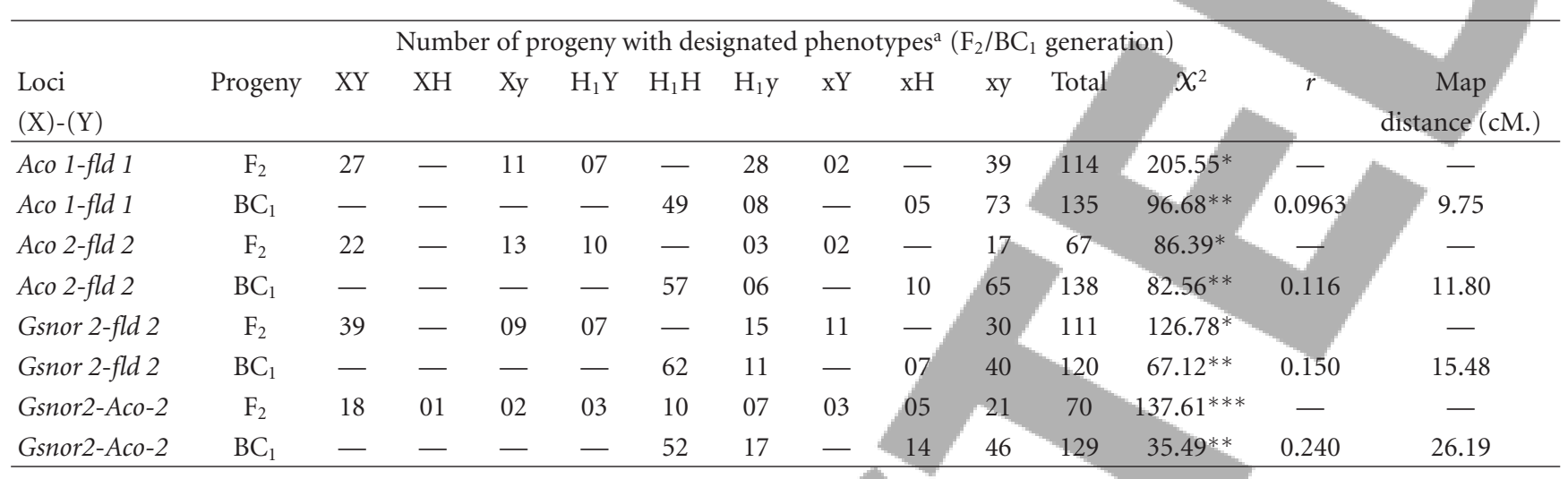

TABLE 4: Segregations of fld 1 and fld 2 along with Aco 1, Aco 2, and Gsnor 2 isozyme loci in $\mathrm{F}_{2}$ and $\mathrm{BC}_{1}$ generations obtained from several crosses (data pooled) between two different primary trisomics (Tr I and IV) and four diploid parents. Data of only critical trisomics carrying isozyme loci presented here. ${ }^{\mathrm{a}} \mathrm{F}$ - Homozygous for fast/dominant allele, S-Homozygous for slow/recessive allele, H-Heterozygous; $(2 n+1)^{\mathrm{b}}$ consistent at 5\% level; *,**, and ${ }^{* *}$ consistent with $4: 4: 1,8: 1$ in $\mathrm{F}_{2}$, and $2: 1$ in $\mathrm{BC}_{1}$ at $5 \%$ level of significance, respectively.

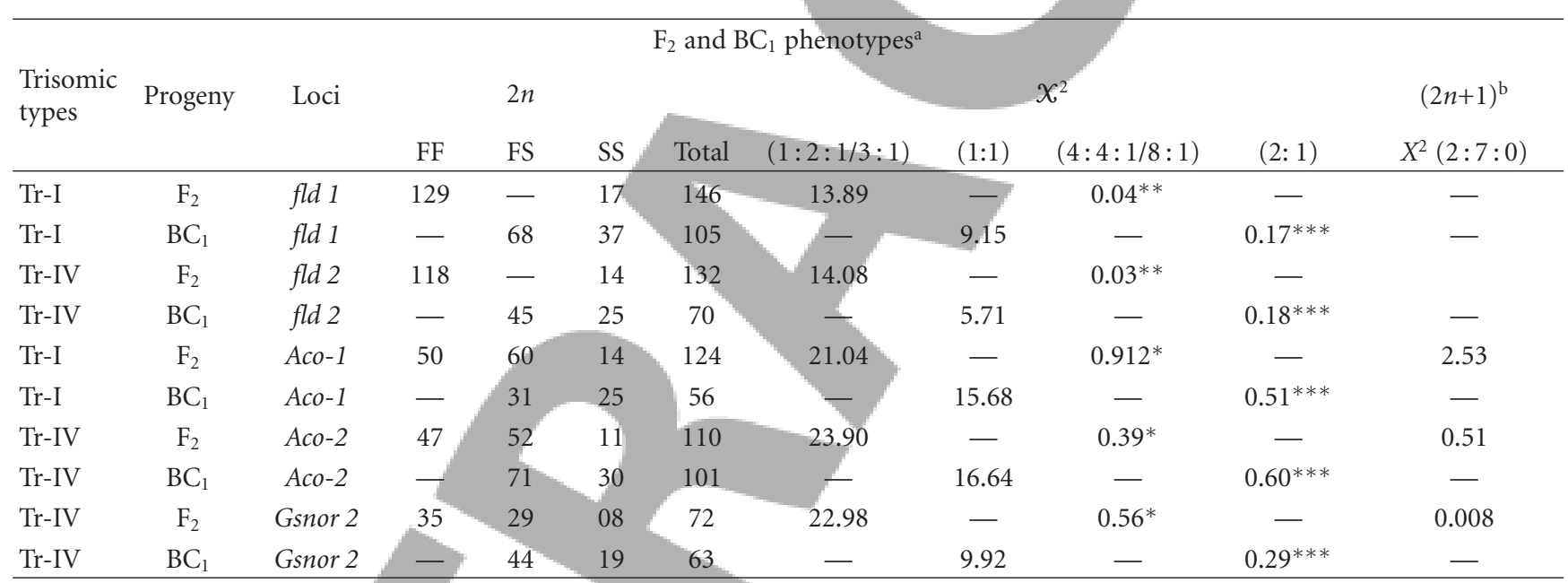

one, designated as ACO-1 contained a total of three bands exhibiting two different types of migration (Figures 1 and 4). The fast moving allozyme (ACO-1a) was unique to bothmutants (lane 1 and 3), whereas relatively slower variant (ACO-1b) was common in mother control variety (lane 2). In the ACO-2 zone, three different types of mobility were manifested by a total of three bands; one of them was fast moving (ACO-2a) and developed only in fldL-1 (lane 1). It was followed by a unique slower band (ACO-2b) generated in fldL-2 mutant (lane 3). The slowest band in this zone (ACO2c) was found specific to mother variety, BioL-212 (Figures 1 and 4, lane 2). The other variety "Hooghly Local" produced identical zymograms of var. BioL-212 (not shown in figure).

Two types of allozyme activity in ACO-1 zone have been confirmed in segregating populations of different $F_{2}$ and backcross (Figures 2 and 5). Segregation of Aco-1a/Aco-1b was found in mutant (Aco 1a/Aco 1a) $\times$ control variety (Aco 1b/Aco 1b) (Table 2). In the Aco 2 locus, heterozygous individuals for the alleles Aco $2 a / A c o 2 b$ were detected in the
$\mathrm{F}_{2}$ progeny of crosses involving fld $L-1$ (Aco 2a/Aco 2a) and fld L-2 (Aco 2b/Aco 2b) mutants. Similarly, crosses between fld L-2 (Aco 2b/Aco 2b) and two control varieties (Aco $2 c / A c o$ 2c) and between fld L-1 (Aco 2a/Aco 2a) and control varieties yielded homozygous individuals parental types and heterozygous individuals for Aco $2 b / A c o$ in former cross and for Aco $2 a / A c o \quad 2 c$ in case of latter. $F_{1}$ phenotype and one parental phenotype were observed in backcross progeny. In case of both loci, three types of phenotypes-two single-banded homozygous parental and one double-banded heterozygous phenotypes of fast and slow allozymes in $\mathrm{F}_{2}$ and two phenotypes: one heterozygous and one respective parental type in corresponding backcrosses segregated and conformed well with $1: 2: 1$ in $\mathrm{F}_{2}$ and $1: 1$ in backcrosses, respectively (Table 2 ).

3.3.2. GSNOR. Two control varieties and two induced mutant lines together generated 4 bands which could be 


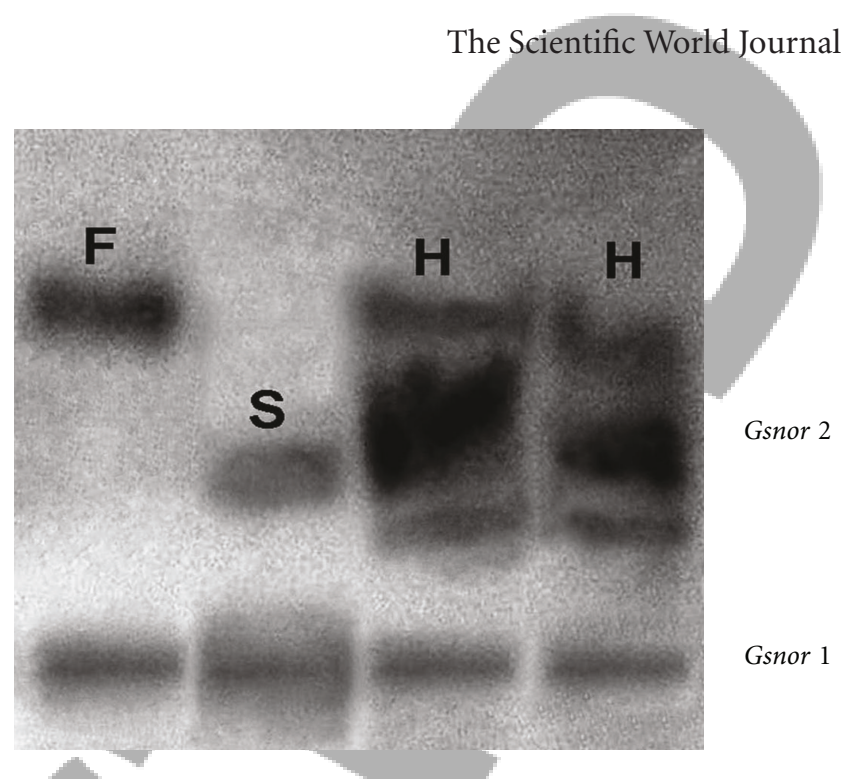

Figure 1: Zymogram phenotype of aconitase isozymes; lane 1fldL-1, lane 2-mother variety BioL-212, lane 3-fldL-2 mutant, small letters indicate alleles of respective loci in Lathyrus sativus $\mathrm{L}$.

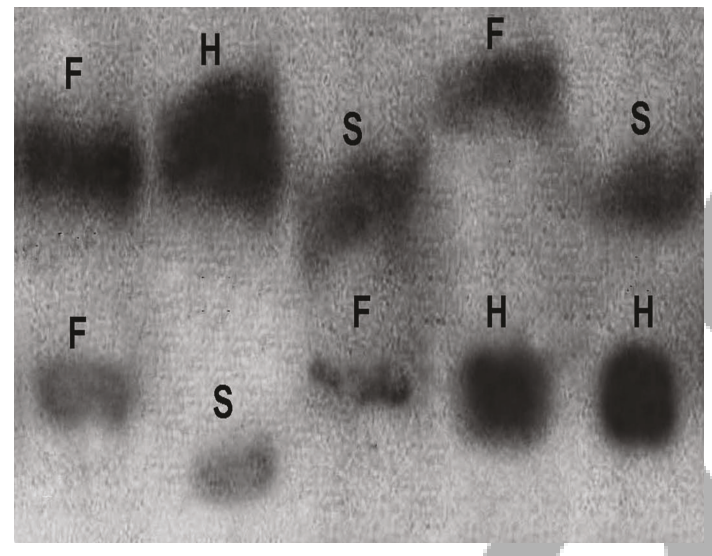

FIGURE 2: Segregation of phenotypes in $\mathrm{F}_{2}$ derived from crosses between BioL-212 $\times$ fldL- 1 in Aco 1 and Aco 2 loci; F-Fast allele, S-slow allele, $\mathrm{H}$-heterozygotes in different lanes, in Lathyrus sativus L.

clearly resolved in two separate zones of enzyme activity tentatively designated as GSNOR1 and GSNOR 2 from anodal side of the gel in the present material. All the four parents bred true in successive selfed $\left(\mathrm{M}_{2}-\mathrm{M}_{4}\right)$ generations for the single-banded pattern corresponding to different allozymes in these two zones, and only representative zymograms showing differences in banding pattern have been shown. The mutant line fld $L-2$ was conspicuously different from control and also from fld $L-1$ by possessing a unique band in zymogram (lane 2). The GSNOR 1 zone was monomorphic with same mobility and intensity of bands in all four parents. By contrast, in GSNOR 2 zone, the fastest band at lane 1 (GSNOR 2a) was present in control variety and fld $L-1$ mutant, while the slower one at lane 2 (GSNOR 2b) was visualized as unique band in fldL-2 line (Figure 3 ).

$\mathrm{F}_{2}$ progeny revealed allelic segregation (single locus) in GSNOR 2 zones of enzyme activity in crosses between fld L-2 mutant and other three parents (Figures 3 and 6). Allozymes in this zone segregated into three phenotypic classes: two
FIgURE 3: Segregation of S-nitrosoglutathione reductase loci, Gsnor 2 in F2 generation of fldL-2 $\times$ mother plant; F-Fast allele, S-slow allele, H-heterozygote. No segregation was observed in Gsnor 1 locus in Lathyrus sativus $\mathrm{L}$.
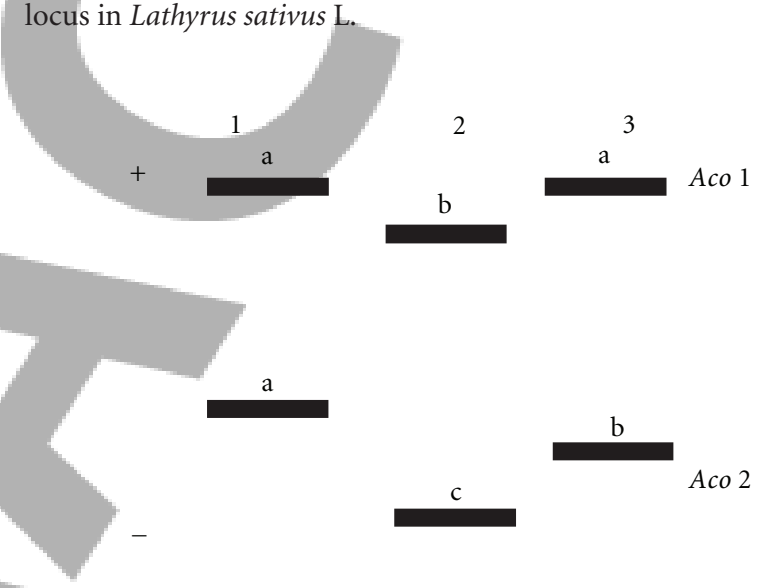

FIGURe 4: Zymogram phenotype of aconitase isozymes; lane 1-fldL1, lane 2-mother variety BioL-212, and lane 3-fldL-2 mutant. Small letters indicate alleles of respective loci in Lathyrus sativus $\mathrm{L}$.

homozygotes for respective parental alleles (lanes 1 and 2) and one heterozygote of these two alleles (lanes 3 and 4) showing good agreement with the expected $1: 2: 1$ ratio in $\mathrm{F}_{2}$ generation (Figures 3 and 6; Table 2), and no segregation distortion was found. The $\mathrm{F}_{1}$ hybrid was backcrossed to parents slowing slow allozyme and zymogram phenotypes agreed well with 1 parental : 1 hybrid ratio in each cross (Table 2). Segregation of allozymes could not be detected in $F_{2}$ progeny of two control varieties in this zone. No segregation of banding pattern was observed in GSNOR 1 zone also.

3.4. Linkage Analysis between Isozyme Loci, fld 1, and fld 2 - Genetic linkage relationship was analyzed on the basis of joint segregation of zymogram phenotype and flavonoid level in $\mathrm{F}_{2}$ and backcrosses (Table 3). Individual locus in Aco and Gsnor loci (except Gsnor 1) as well as fld 1 and fld 2 exhibited normal mendelian segregation $\left(1: 2: 1 / 3: 1\right.$ in $F_{2}$ and $1: 1$ in backcrosses) of alleles, but their joint segregation 


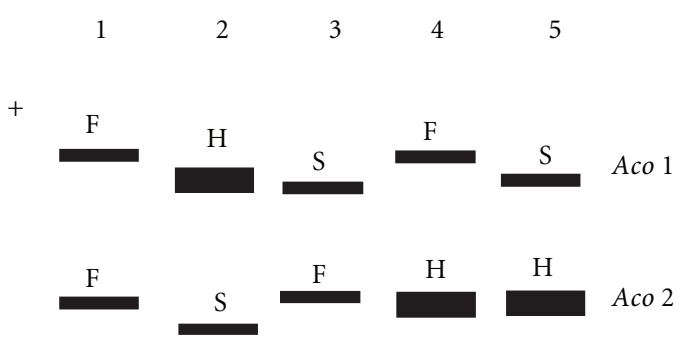

Figure 5: Segregation of phenotypes in $\mathrm{F}_{2}$ derived from crosses between BioL-212 $\times$ fldL-1 in Aco 1 and Aco 2 loci; F-Fast allele, $\mathrm{S}$-slow allele, H-heterozygotes in different lanes, in Lathyrus sativus L.

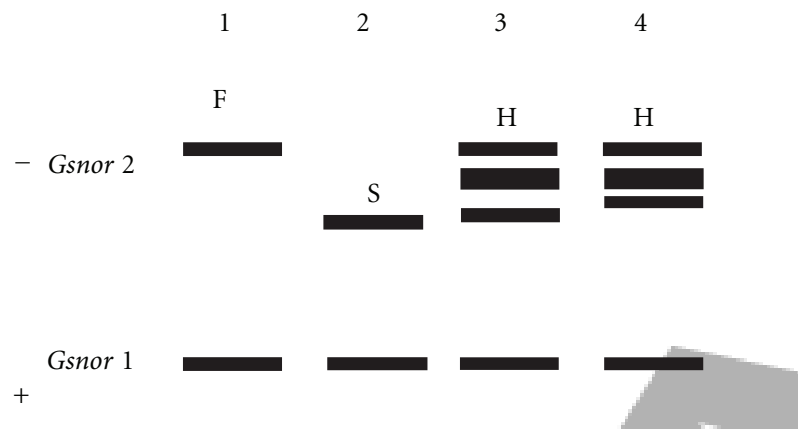

FIGURE 6: Segregation of S-nitrosoglutathione reductase loci, Gsnor 2 in $\mathrm{F}_{2}$ generation of fldL- $2 \times$ mother plant; F-Fast allele, S-slow allele, H-heterozygote. No segregation was observed in Gsnor 1 locus in Lathyrus sativus L.

in different cross-combinations showed significant deviations $(P<0.05)$ from the expected ratios of independent assortment in different $\mathrm{F}_{2}$ families and respective backcross progenies (Table 3 ). In each case, recombination fraction $(r)$ calculated from backcross data was put into Kosambi's mapping function, and map distance between loci was estimated. Aco 1 and fld 1 was linked with a map distances of $9.75 \mathrm{cM}$, whereas fld 2 and Aco 2 were mapped $11.80 \mathrm{cM}$ apart. A linked association with $15.48 \mathrm{cM}$ and $26.19 \mathrm{cM}$ map distance was found between fld 2 and Gsnor 2 and between Aco 2 and Gsnor 2, respectively (Table 3). Gsnorl could not be mapped due to absence of segregating alleles.

3.5. Chromosome Location of fld1, fld2, and Isozyme Loci by Primary Trisomic Analysis. Chromosomal association of fld1 and fld 2 loci controlling different phenotypes of flavonoid deficiency in grass pea was traced in crosses between seven trisomics as female parents and the diploid mutant lines as their male counterpart. For trisomic-I and IV, the segregation of leaf flavonoid content as normal, recessive mutant phenotype derived from fldL-1 $\times$ trisomic-I and from fldL- $2 \times$ trisomic-IV, respectively, exhibited a large and significant $X^{2}$ value $(P<0.05)$ for $3: 1$ in $\mathrm{F}_{2}$ and $1: 1$ in backcrosses but agreed well with expected trisomic ratios of $8: 1$ in $\mathrm{F}_{2}$ and $2: 1$ in testcross progenies (Table 4). On the other hand, segregation of normal and recessive mutant type in rest of the crosses involving other trisomics showed good fit with expected disomic ratio of $3: 1$ ratio in F2 and $1: 1$ ratio in corresponding testcrosses in diploid population, and a good number of recessive homozygotes in $2 n+1$ portion of these crosses were cytologically detected as trisomic plants (data not in table).

Among the four isozyme loci visualized in gel, linkage was detected only between Aco 2 and Gsnor 2. Presumably, these two isozyme loci were on the same chromosome. To confirm this assumption and to localize them on chromosomes, the trisomics were crossed as female parent with diploid control and two mutant lines, and $\mathrm{F}_{1}$ progeny in each case was raised. The rationale of the trisomic analysis in the present material involved trisomic segregation of different phenotypes in diploid portion of $\mathrm{F}_{2}$ and $\mathrm{BC}_{1}$. A significant $(P<0.05)$ departure of allozyme segregation coded by Aco 1 from normal disomic ratios in $\mathrm{F}_{2}(1: 2: 1)$ as well as backcross $(1: 1)$ ratios was manifested in the progenies involving only trisomic-I. Similar situation was encountered for Aco 2 and Gsnor 2 loci in trisomic-IV (Table 4). For both cases, segregation of allozymes in respective trisomics agreed well with the expected trisomic ratio of $4: 4: 1$ in $\mathrm{F}_{2}$ and to $2: 1$ in $B_{1}$ generations of diploid portion and to $2: 7: 0$ $\left(\mathrm{F}_{2}\right)$ in trisomic portion of the progeny (Table 4). Segregation was disomic for all other trisomics in $\mathrm{F}_{2}$ and corresponding backcrosses (data not presented).

Linkage studies and trisomic segregation pattern in $\mathrm{F}_{2}$ as well as $\mathrm{BC}_{1}$ generations revealed that Acol and fld 1loci were linked with each other on extra chromosome of trisomicI, whereas fld 2 and two isozyme loci Aco 2 and Gsnor 2 were carried by extra chromosome of trisomic-IV in linked conditions. Based on the result, the map positions (in cM) among different loci are as shown in Figure 7.

\section{Discussion}

Both fldL-1 and fldL-2 mutants, isolated in EMS-treated $\mathrm{M}_{2}$ progeny, exhibited huge deficiency in total foliar flavonoid contents, containing only $20 \%$ of normal level as measured in mother controls. However, the mutants differed from each other in the type of extract, where the flavonoid content reduced, ethanol extract for $f l d L-1$ and aqueous extract for fldL-2 leaves. Flavonoid deficiency was found associated with modification of usual blue colour of flower into white and stipule characters in both type of mutants, and also rising level of pollen sterility in fldL-2 plants. The modification of flower colour was ascribed to the deficiency of anthocyanin biosynthesis and is of considerable assistance in plant breeding [45]. In Petunia, flavonoid deficiency resulted in male sterility [46], while in maize male fertility was not affected at all [47]. Both the phenomena, however, were found in the present mutants, supporting differential behavior of the two mutants and deficiency of total flavonoids might be due to reduction of ethanol-dissolved and water-soluble compounds. Flavonoid deficiency in Arabidopsis was found associated with modifications in seed testa colour [7] and UV-sensitivity [6]. Both fldL-1 and fldL-2 in the present study 
Aco 2----11.80----fld 2----15.48----Gsnor 2

I------------------26.19-------------------I

Figure 7

are nonlethal and provided easily detectable phenotypes such as flower color. No significant variation of flavonoid content, however, was found in two mother plants, suggesting lack of its variation in common genotypes of grass pea.

Mode of inheritance of flavonoid deficiency was traced in self-pollinated as well as in intercrossed population, involving two mutants and two mother controls. In all four parents, marginal variation in flavonoid content was found in advanced generations, indicating true breeding nature of the mutant traits. Inheritance studies in intercrossed progeny obtained from control $\times$ mutant plants revealed monogenic recessive nature of the low flavonoid content in both the mutant types with dominant allele that was always with mother plants. The result is in agreement with monogenic recessive nature of different flavonoid mutants in plants including Arabidopsis [7]. Interestingly, flower colour and stipule characteristics appeared unmodified in the respective recessive mutant type, confirming their true breeding nature in the present material.

A completely different result, however, was obtained when the two mutants were crossed reciprocally. Occurrence of $\mathrm{F}_{1}$ plants with normal level of flavonoids and usual presence of blue flower and papilionaceous stipules and its segregations into four different plant types: normal, fldL-1, fldL-2, and a double-mutant type, consistent with $9: 3: 3: 1$ ratio in $F_{2}$, suggested involvement of two independent nonallelic loci Fld1/fld 1 (for fldL-1 mutant) and Fld2/fld 2 (for fld L-2 mutant) in controlling flavonoid deficiency in two mutant types under study. Both the genes (Fld 1 and Fld 2) exhibited dominance over their respective recessive alleles (fld1 and fld 2). In presence of both the genes in dominant form (Fld 1-Fld 2-), normal phenotype appeared whereas presence of fld 2 gene in double recessive form (fld 2 fld 2 Fld 2-) produced phenotypes characteristic of fldL-2 type. On the other hand, fldL-1 type occurred in the presence of double recessive nature of fld 1 gene (Fld1fld 1 fld 1 ). In homozygous recessive condition of both the genes (fld 1 fld 1 fld 2 fld 2 ) variant plant type showing leaf flavonoid content only $10 \%$ of mother control plants and high pollen sterility $(79.33 \%)$ resulted in the $\mathrm{F}_{2}$ progeny. This type bred true in advanced generations and tentatively designated as "flavonoid-deficient double mutant type" in grass pea. Recovery of $f l d L-1$ and fldL-2 phenotypes in $\mathrm{F}_{2}$ and occurrence of the double mutant type strongly indicated possibility of multiple blockages in flavonoid biosynthesis pathway which was different in two mutant types, but combined in double mutant plants, leading to further depletion of its flavonoid content in relation to fldL-1 and fldL-2 levels. The double mutants have immense significance as it provides valuable clues in functional biology of glutathione, NO and thioredoxin-mediated redox signaling in plants $[14,48]$.
The differences in genetic constitution of flavonoid deficiency between two mutant plant types were also manifested by banding profiles of aconitase and S-glutathione reductase isozymes. Inheritance pattern in the present study revealed that fldL-1 and fldL-2 were not only different from control varieties but also differed from each other due to variant banding profiles that were heritable and bred true for all the four loci resolved here. The distinct zones of enzyme activity are mostly coded by different loci, and the variants within a particular zone are usually due to presence of different alleles or their interaction as heterozygotes [43]. In the present material, consistency in two zones of enzyme activity for both ACO and GSNOR enzymes was confirmed in successive selfpollinated and intercrossed populations of four parents.

Quite remarkably, the "loss-of-function" mutation in flavonoid content led to gain of isozyme functions in the present mutants. Allozyme variation is essential for construction of saturated linkage map with other markers in grass pea $[35,36]$. Although control varieties showed monomorphic banding pattern, consistent occurrences of mutant specific bands in the present Aco and Gsnor loci indicated evolution of variant alleles which inherited as recessive gene mutations in the present material of grass pea. Both the mutant lines possessed some unique bands coded by specific alleles: Aco $2 a$ in fld $L-1$ and Gsnor $2 b$ and Aco $2 b$ in fld L-2. Obviously, Aco 2 was triple allelic while Aco 1 and Gsnor 2 both were double allelic, resulting in increased polymorphism in the present mutants over their control plants. Presence of more than two alleles was also reported in Aco loci of grass pea [35] and lima bean [49]. Like Gsnor 1 , single zone of activity was reported in GSNOR enzyme of Pisum sativum L. [15].

Segregation pattern of different allozymes in the present $\mathrm{F}_{2}$ and backcross-population indicated involvement of codominant alleles in monogenic segregation of Aco 1, Aco 2 loci of ACO system, and Gsnor 2 locus of GSNOR enzyme. Presence of double-banded phenotypes in heterozygotes suggested monomeric nature of aconitase in grass pea, and no distorted segregation was apparent in $\mathrm{F}_{2}$ generation. The GSNOR, on the other hand, was functionally dimeric as confirmed by the presence of four-banded phenotypes in the heterozygotes. However, single-banded phenotype was exhibited in heterozygotes of $\mathrm{F}_{2}$ and backcross-populations obtained from crosses involving mutant and control parents for Gsnor 1 locus, confirming its monomorphic nature in the present material. In $\mathrm{F}_{2}$ population of crosses between different subaccessions of Lathyrus sativus L., Chowdhury and Slinkard [35] also detected polymorphism in both Aco 1 and Aco 2 loci with occurrence of codominant alleles, while, in regenerated plants of soybean, a rare mutation in $A c o 2 b$ locus was detected as a null allele [50]. Polymorphisms displaying segregation ratios close to those expected for single 
locus traits suggested involvement of different alleles at the structural loci in generation of variation in different isozyme loci [51]. Among the closely related genera of Lathyrus, Aco 1 was monomorphic, but Aco 2 was polymorphic in Vicia faba L. [52]. Polymorphic Aco loci showing codominant expression of different alleles were also studied in pea [43], lens [53], and Cicer arietinum L. [54, 55].

Absence of distortion in $\mathrm{F}_{2}$ single locus and joint segregation was another interesting feature in the present study consisting of four true breeding parental lines of Lathyrus sativus $\mathrm{L}$. for the concerned traits. The result was in contrast with earlier reports of distorted segregation of other isozyme loci in grass pea [35]. Helentjaris et al. [56] explained that intraspecific cross-minimized genetic distortion and other errors than wide crosses to establish linkage maps. The true breeding nature of isozyme phenotypes in selfed progeny and simple segregation in $\mathrm{F}_{2}$ population of different intercrossed progenies confirmed their stability in the present material.

Mutation has been identified as one of the main sources of isozyme variation in higher plants [57]. For the first time, allelic variations in Aco and Gsnor loci have been generated in two stable mutant lines, deficient in flavonoid contents, of grass pea through induced mutagenesis. Consistent presence of polymorphism in isozymes of both enzymes indicated origin of different molecular forms of allozymes. Induction of variant allele in leaf isozyme system has been reported in different legumes including Glycine max [50] and Trifolium resupinatum [58]. However, in some accessions of Lathyrus sativus L. and Centrosema occurrences of higher number of alleles per locus have been attributed to heterozygosity induced by significant outcrossing rate in these crops [36]. In the present study, in addition to using outcrossing preventive measure during hybridization, effective isolation between lines and populations has been maintained throughout the experiment to prevent intermixing, and inheritance studies were carried out in advanced selfed generation $\left(\mathrm{M}_{4}\right)$ of different true breeding parental lines. It seemed likely that the occurrences of new alleles in Aco and Gsnor loci resulted from the action of the recessive genes induced by EMS treatments in the present materials.

Linkage analysis involving Aco 1, Aco 2, and Gsnor 2 isozyme loci, and fld 1 and fld 2 mutations revealed independent assortment between two Aco loci, of which Aco 1 was linked tightly with fld 1 whereas Aco 2 was mapped with fld 2 and Gsnor 2 loci in linked states, showing a distance of $11.80 \mathrm{cM}$ and $26.19 \mathrm{cM}$, respectively. Absence of linkage between different Aco loci was also reported in different genotypes of grass pea, soybean, and Phaseolus vulgaris $[35,59]$, and this was confirmed in the present study also. However, for the first time, a Gsnor locus was mapped in linked association with a flavonoid-deficient locus and also with an Aco locus in any leguminous crop. The aconitase is exquisitely sensitive to NO and other ROS [17], while Gsnor reportedly showed reduced band intensity in cadmium-treated Pisum sativum L. [15]. Altered expression of the present Aco and Gsnor loci indicated modulation of enzyme activities under flavonoid-deficient conditions, and the mapping of their isozyme loci with fld 1 and fld 2 genes in closely linked state confirmed this assumption.
The importance of any mutant trait as a potential tool in functional biology enhanced once it was assigned to a particular chromosome. Primary trisomic has been used as an excellent tool in legume crops to confirm possible chromosomal location of various traits [60]. When the loci under study were located on a particular chromosome in trisomy, the normal disomic segregation ratio was modified due to presence of an extra chromosome. Trisomic segregation of electrophoretic phenotypes of different isozymes in the present zymogram strongly indicated possible location of Aco 1 on extra chromosome of trisomic-I and Aco 2 and Gsnor 2 on extra chromosome of trisomic-IV. Similarly, a good fit of fld 1 and fld 2 to trisomic segregation strongly indicated possible location of fld 1 gene on extra chromosome of trisomic I and that of fld 2 gene on extra chromosome of trisomic IV. The deviations from the normal segregation ratio are ascribed to the phenomenon of primary trisomy. Furthermore, no recessive homozygote plant in trisomic portion was recovered in these crosses, and all the recessive homozygotes in population were cytologically confirmed as diploids (data not presented). Segregating phenotypes in $\mathrm{F}_{2}$ and $\mathrm{BC}_{1}$ generations involving other trisomic types in respective crosses were consistent with normal mendelian disomic ratios and confirmed the above observation. In grass pea, primary trisomic has been successfully utilized to assign genes of agronomic interest on specific chromosomes $[21,24,61]$ and to study gene-dosage effect of aneuploidy on antioxidant defense enzymes [62]. The isolation of two different flavonoid-deficient mutants and their mapping with closely linked isozyme markers of two prominent enzymatic systems on specific chromosomes may provide vital clues in understanding the role of flavonoid in integrated antioxidant defense system and their genetic basis in grass pea.

\section{Conflict of Interests}

No conflict of interests is involved in any way with the present work.

\section{References}

[1] L. P. Taylor and E. Grotewold, "Flavonoids as developmental regulators," Current Opinion in Plant Biology, vol. 8, no. 3, pp. 317-323, 2005.

[2] Y. Miyagi, A. S. Om, K. M. Chee, and M. R. Bennink, "Inhibition of azoxymethane-induced colon cancer by orange juice," Nutrition and Cancer, vol. 36, no. 2, pp. 224-229, 2000.

[3] T. A. Holton and E. C. Cornish, "Genetics and biochemistry of anthocyanin biosynthesis," Plant Cell, vol. 7, no. 7, pp. 10711083, 1995.

[4] S. Chopra, A. Hoshino, J. Boddu, and S. Iida, "Flavonoid pigments as tools in molecular genetics," in The Science of Flavonoids, E. Grotewold, Ed., pp. 147-173, The Ohio State University, Columbus, Ohio, USA, 2006.

[5] R. Koes, W. Verweij, and F. Quattrocchio, "Flavonoids: a colorful model for the regulation and evolution of biochemical pathways," Trends in Plant Science, vol. 10, no. 5, pp. 236-242, 2005. 
[6] J. Li, T. M. Ou-Lee, R. Raba, R. G. Amundson, and R. L. Last, "Arabidopsis flavonoid mutants are hypersensitive to UV-B irradiation," Plant Cell, vol. 5, no. 2, pp. 171-179, 1993.

[7] B. W. Shirley, W. L. Kubasek, G. Storz et al., "Analysis of Arabidopsis mutants deficient in flavonoid biosynthesis," Plant Journal, vol. 8, no. 5, pp. 659-671, 1995.

[8] S. Albert, M. Delseny, and M. Devie, "Banyuls, a novel negative regulator of flavonoid biosynthesis in the Arabidopsis seed coat," Plant Journal, vol. 11, no. 2, pp. 289-299, 1997.

[9] K. Bieza and R. Lois, "An Arabidopsis mutant tolerant to lethal ultraviolet-B levels shows constitutively elevated accumulation of flavonoids and other phenolics," Plant Physiology, vol. 126, no. 3, pp. 1105-1115, 2001.

[10] M. Sharma, M. Cortes-Cruz, K. R. Ahern, M. McMullen, T. P. Brutnell, and S. Chopra, "Identification of the Pr1 gene product completes the anthocyanin biosynthesis pathway of maize," Genetics, vol. 188, no. 1, pp. 69-79, 2011.

[11] R. A. Dixon and L. W. Sumner, "Legume natural products: understanding and manipulating complex pathways for human and animal health," Plant Physiology, vol. 131, no. 3, pp. 878-885, 2003.

[12] J. Filkowski, O. Kovalchuk, and I. Kovalchuk, "Genome stability of vtc1, tt4, and tt5 Arabidopsis thaliana mutants impaired in protection against oxidative stress," Plant Journal, vol. 38, no. 1, pp. 60-69, 2004.

[13] M. R. Alfenito, E. Souer, C. D. Goodman et al., "Functional complementation of anthocyanin sequestration in the vacuole by widely divergent glutathione $S$-transferases," Plant Cell, vol. 10, no. 7, pp. 1135-1149, 1998.

[14] T. Bashandy, L. Taconnat, J.-P. Renou, Y. Meyer, and J.P. Reichheld, "Accumulation of flavonoids in an ntra ntrb mutant leads to tolerance to UV-C," Molecular Plant, vol. 2, no. 2, pp. 249-258, 2009.

[15] J. B. Barroso, F. J. Corpas, A. Carreras et al., "Localization of $S$-nitrosoglutathione and expression of $S$-nitrosoglutathione reductase in pea plants under cadmium stress," Journal of Experimental Botany, vol. 57, no. 8, pp. 1785-1793, 2006.

[16] W. Moeder, O. Del Pozo, D. A. Navarre, G. B. Martin, and D. F. Klessig, "Aconitase plays a role in regulating resistance to oxidative stress and cell death in Arabidopsis and Nicotiana benthamiana," Plant Molecular Biology, vol. 63, no. 2, pp. 273287, 2007.

[17] D. A. Navarre, D. Wendehenne, J. Durner, R. Noad, and D. F. Klessig, "Nitric oxide modulates the activity of tobacco aconitase," Plant Physiology, vol. 122, no. 2, pp. 573-582, 2000.

[18] A. Sakamoto, M. Ueda, and H. Morikawa, "Arabidopsis glutathione-dependent formaldehyde dehydrogenase is an Snitrosoglutathione reductase," FEBS Letters, vol. 515, no. 1-3, pp. 20-24, 2002.

[19] M. Díaz, H. Achkor, E. Titarenko, and M. C. Martínez, "The gene encoding glutathione-dependent formaldehyde dehydrogenase/GSNO reductase is responsive to wounding, jasmonic acid and salicylic acid," FEBS Letters, vol. 543, no. 1-3, pp. 136-139, 2003.

[20] E. Pastor-Cavada, R. Juan, J.E. Pastor, J. Girón-Calle, M. Alaiz, and J. Vioque, "Antioxidant activity in Lathyrus species," Grain Legumes, vol. 54, pp. 10-11, 2009.

[21] D. Talukdar, "Dwarf mutations in grass pea (Lathyrus sativus L.): Origin, morphology, inheritance and linkage studies," Journal of Genetics, vol. 88, no. 2, pp. 165-175, 2009.

[22] D. Talukdar and A. K. Biswas, "Inheritance of flower and stipule characters in different induced mutant lines of grass pea (Lathyrus sativus L.)," Indian Journal of Genetics and Plant Breeding, vol. 67, pp. 396-400, 2007.
[23] D. Talukdar and A. K. Biswas, "Induced seed coat colour mutations and their inheritance in grass pea (Lathyrus sativus L.)," Indian Journal of Genetics and Plant Breeding, vol. 65, pp. 135136, 2005.

[24] D. Talukdar, "Genetics of pod indehiscence in Lathyrus sativus L," Journal of Crop Improvement, vol. 25, pp. 1-15, 2011.

[25] D. Talukdar, "Bold-seeded and seed coat colour mutations in grass pea (Lathyrus sativus L.): origin, morphology, genetic control and linkage analysis," International Journal of Current Research, vol. 3, pp. 104-112, 2011.

[26] D. Talukdar, "Flower and pod production, abortion, leaf injury, yield and seed neurotoxin levels in stable dwarf mutant lines of grass pea (Lathyrus sativus L.) differing in salt stress responses," International Journal of Current Research, vol. 2, pp. 46-54, 2011.

[27] D. Talukdar, "Isolation and characterization of NaCl-tolerant mutations in two important legumes, Clitoria ternatea L. and Lathyrus sativus L.: Induced mutagenesis and selection by salt stress," Journal of Medicinal Plant Research, vol. 5, no. 16, pp. 3619-3628, 2011.

[28] D. Talukdar, "Effect of arsenic-induced toxicity on morphological traits of Trigonella foenum-graecum L. and Lathyrus sativus L during germination and early seedling growth," Current Research Journal of Biological Sciences, vol. 3, pp. 116123, 2011.

[29] D. Talukdar, "Ascorbate deficient semi-dwarf asfL1 mutant of grass pea (Lathyrus sativus L.) exhibits alterations in antioxidant defense," Biologia Plantarum. In press.

[30] D. Talukdar and A. K. Biswas, "Seven different primary trisomics in grass pea (Lathyrus sativus L.). I. Cytogenetic characterisation," Cytologia, vol. 72, no. 4, pp. 385-396, 2007.

[31] D. Talukdar, "Cytogenetic characterization of seven different primary tetrasomics in grass pea (Lathyrus sativus L.)," Caryologia, vol. 61, no. 4, pp. 402-410, 2008.

[32] D. Talukdar, "Cytogenetic characterization of induced autotetraploids in grass pea (Lathyrus sativus L.)," Caryologia, vol. 63, no. 1, pp. 62-72, 2010.

[33] D. Talukdar, "Reciprocal translocations in grass pea (Lathyrus sativus 1.): pattern of transmission, detection of multiple interchanges and their independence," Journal of Heredity, vol. 101, no. 2, pp. 169-176, 2010.

[34] D. Talukdar, "Cytogenetic analysis of a novel yellow flower mutant carrying a reciprocal translocation in grass pea (Lathyrus sativus L.)," Journal of Biological Research-Thessaloniki, vol. 15, pp. 123-134, 2011.

[35] M. A. Chowdhury and A. E. Slinkard, "Genetics of isozymes in grasspea," Journal of Heredity, vol. 91, no. 2, pp. 142-145, 2000.

[36] J. F. Gutiérrez, V. Francisca, and J. V. Francisco, "Genetic mapping of isozyme loci in Lathyrus sativus L," Lathyrus Lathyrism Newsletter, vol. 2, pp. 74-78, 2001.

[37] D. Talukdar, "Allozyme variations in leaf esterase and root peroxidase isozymes and linkage with dwarfing genes in induced dwarf mutants of grass pea (Lathyrus sativus L.)," International Journal of Genetics and Molecular Biology, vol. 2, no. 6, pp. 112-120, 2010.

[38] D. Talukdar and A. K. Biswas, "Seven different primary trisomics in grass pea (Lathyrus sativus L.). II. Pattern of transmission," Cytologia, vol. 73, no. 2, pp. 129-136, 2008.

[39] N. Wu, K. Fu, Y.-J. Fu et al., "Antioxidant activities of extracts and main components of pigeonpea [Cajanus cajan (L.) Millsp.] leaves," Molecules, vol. 14, no. 3, pp. 1032-1043, 2009.

[40] B. J. Cardy, C. W. Stuber, and M. M. Goodman, Techniques for starch gel electrophoresis of enzymes from maize (Zea mays L.), 
Institute of Statistics, Mimeograph Series No. 1317, N.C. State University, Raleigh, NC, USA, 1980.

[41] B. J. Cardy and W. D. Beversdorf, "A procedure for the starch gel electrophoretic detection of isozymes of soybean (Glycine max [L] Merr.)," Technical Bulletin, no. 119/8401, Department of Crop Science, University of Guelph, Guelph, Canada, 1984.

[42] M. R. Fernández, J. A. Biosca, and X. Parés, "S-nitrosoglutathione reductase activity of human and yeast glutathionedependent formaldehyde dehydrogenase and its nuclear and cytoplasmic localisation," Cellular and Molecular Life Sciences, vol. 60, no. 5, pp. 1013-1018, 2003.

[43] N. F. Weeden, "A suggestion for the nomenclature of isozyme loci," Pisum Newsletter, vol. 20, pp. 44-45, 1988.

[44] D. D. Kosambi, "The estimation of map distance from recombination values," Annals of Eugenics, vol. 12, pp. 172175, 1944.

[45] R. A. Dixon and N. L. Paiva, "Stress-induced phenylpropanoid metabolism," Plant Cell, vol. 7, no. 7, pp. 1085-1097, 1995.

[46] I. M. Van Der Meer, M. E. Stam, A. J. Van Tunen, J. N. M. Mol, and A. R. Stuitje, "Antisense inhibition of flavonoid biosynthesis in petunia anthers results in male sterility," Plant Cell, vol. 4, no. 3, pp. 253-262, 1992.

[47] Y. Mo, C. Nagel, and L. P. Taylor, "Biochemical complementation of chalcone synthase mutants defines a role for flavonols in functional pollen," Proceedings of the National Academy of Sciences of the United States of America, vol. 89, no. 15, pp. 7213-7217, 1992.

[48] A. Shahpiri, B. Svensson, and C. Finnie, "The NADPHdependent thioredoxin reductase/thioredoxin system in germinating barley seeds: gene expression, protein profiles, and interactions between isoforms of thioredoxin $h$ and thioredoxin reductase," Plant Physiology, vol. 146, no. 2, pp. 789-799, 2008.

[49] I. Zoro Bi, A. Maquet, B. Wathelet, and J.-P. Baudoin, "Genetic control of isozymes in the primary gene pool of Phaseolus lunatus L," Biotechnology, Agronomy, Society and Environment, vol. 3, pp. 10-27, 1999.

[50] L. A. Amberger, R. C. Shoemaker, and R. G. Palmer, "Inheritance of two independent isozyme variants in soybean plants derived from tissue culture," Theoretical and Applied Genetics, vol. 84, no. 5-6, pp. 600-607, 1992.

[51] B. Wolko and N. F. Weeden, "Additional markers for chromosome 6," Pisum Newsletter, vol. 22, pp. 71-74, 1990.

[52] B. Román, Z. Satovic, D. Pozarkova et al., "Development of a composite map in Vicia faba, breeding applications and future prospects," Theoretical and Applied Genetics, vol. 108, no. 6, pp. 1079-1088, 2004.

[53] D. Zamir and G. Ladizinsky, "Genetics of allozyme variants and linkage groups in lentil," Euphytica, vol. 33, no. 2, pp. 329 $336,1984$.

[54] P. M. Gaur and A. E. Slinkard, "Genetic control and linkage relations of additional isozyme markers in chick-pea," Theoretical and Applied Genetics, vol. 80, no. 5, pp. 648-656, 1994.

[55] K. Kazan, F. J. Muehlbauer, N. E. Weeden, and G. Ladizinsky, "Inheritance and linkage relationships of morphological and isozyme loci in chickpea (Cicer arietinum L.)," Theoretical and Applied Genetics, vol. 86, no. 4, pp. 417-426, 1993.

[56] T. Helentjaris, M. Slocum, S. Wright, A. Schaefer, and J. Nienhuis, "Construction of genetic linkage maps in maize and tomato using restriction fragment length polymorphisms," Theoretical and Applied Genetics, vol. 72, no. 6, pp. 761-769, 1986.

[57] Z. Bartošová, B. Obert, T. Takáč, A. Kormuták, and A. Pretová, "Using enzyme polymorphism to identify the gametic origin of flax regenerants," Acta Biologica Cracoviensia Series Botanica, vol. 47, no. 1, pp. 173-178, 2005.

[58] D. R. Malaviya, A. K. Roy, A. Tiwari, P. Kaushal, and B. Kumar, "In vitro callusing and regeneration in Trifolium resupinatum - a fodder legume," Cytologia, vol. 71, no. 3, pp. 229-235, 2006.

[59] B. R. Hedges and R. G. Palmer, "Tests of linkage of isozyme loci with five primary trisomics in soybean, Glycine max (L.) Merr," Journal of Heredity, vol. 82, no. 6, pp. 494-496, 1991.

[60] R. J. Singh, G. H. Chung, and R. L. Nelson, "Landmark research in legumes," Genome, vol. 50, no. 6, pp. 525-537, 2007.

[61] D. Talukdar, "Recent progress on genetic analysis of novel mutants and aneuploid research in grass pea (Lathyrus sativus L.)," African Journal of Agricultural Research, vol. 4, no. 13, pp. 1549-1559, 2009.

[62] D. Talukdar, "The aneuploid switch: Extra-chromosomal effect on antioxidant defense through trisomic shift in Lathyrus sativus L.," Indian Journal of Fundamental and Applied Life Sciences, vol. 1, no. 4, pp. 263-273, 2011. 